\title{
Flexible Performance Debugging of Parallel and Distributed Applications
}

\author{
Jacques Chassin de Kergommeaux ${ }^{1}$, Cyril Guilloud ${ }^{2}$, and B. de Oliveira Stein ${ }^{3}$ \\ 1 Laboratoire LSR-IMAG, B.P. 72, 38402 St. Martin d'Hères Cedex, France \\ Jacques.Chassin-de-Kergommeaux@imag.fr, http://drakkar.imag.fr/ ${ }^{\sim}$ chassin \\ 2 Laboratoire ID-IMAG, ENSIMAG Antenne de Montbonnot \\ ZIRST, 51 avenue Jean Kuntzmann, 38330 Montbonnot Saint Martin, France \\ Cyril.Guilloud@imag.fr, http://www-id.imag.fr/ guilloud \\ 3 Departamento de Eletrônica e Computação, \\ Universidade Federal de Santa Maria - RS, Brazil. \\ benhur@inf.UFSM.br
}

\begin{abstract}
The Pajé approach to help performance debugging of parallel and distributed applications is to provide behavioral visualizations of their program executions to programmers. This article describes how Pajé was designed to be usable for a variety of programming models. Trace recording and trace manipulation facilities can be generated from a description of the events to be traced. The visualization tool includes a trace-driven simulator reconstructing the behavior of the observed executions of the applications being debugged. The Pajé simulator is generic and can be specialized by the types of the objects to be visualized, the representation of these objects and their relations.
\end{abstract}

\section{Introduction}

Performance debugging requires that programmers of parallel and distributed applications identify the bottlenecks, overheads or out of balance computations resulting in inefficient use of parallel resources by their programs. Tools supporting the identification of such problems should help programmers understand the runtime behavior of their applications. Of particular interest are behavioral visualization tools which ease the understanding of the interactions of processes and/or threads during parallel and distributed executions. Behavioral visualization tools reconstruct an execution of a debugged application from execution traces. Such tools need to adapt easily to the variety and continuous evolution of programming models and visualization techniques.

This article describes the Pajé tracing and visualization tools, aimed at easing performance debugging of parallel and distributed applications. These tools adapt to a variety of programming models. Traces are recorded by tracing functions, inserted in the observed applications and automatically generated by "Tumit1" from a description of the events to be recorded. The Pajé visualization

\footnotetext{
${ }^{1}$ Inuit word meaning animal track, track and trace being the same word in French. 
tool is generic, allowing users to describe what they would like to see and how this should be visualized.

The next section discusses the notion of behavioral observation of executions of parallel and distributed applications. The following gives a brief overview of the user-guided generation of trace monitoring and manipulation functions. The genericity of the Pajé visualization tool is then briefly recalled and exemplified. The two last sections survey rapidly related work and conclude the article.

\section{Behavioral Observation of Parallel and Distributed Executions}

\subsection{Monitoring Techniques}

Most monitoring tools are either clock driven or event driven [4] (see figure 1). Clock driven monitoring amounts to have the state of the observed system registered at periodical time intervals. This technique may be useless to identify the origins of some overheads of parallel programs: bottlenecks, communication or idling times. Event driven monitoring is

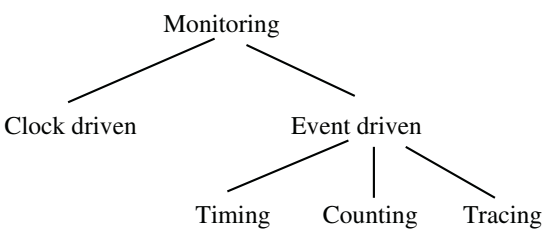

Fig. 1. Classification of monitoring tools

triggered by the advent of observable events. In the timing approach, the time spent in various parts of the observed program is measured. In the counting approach, the number of occurrences of the observed events is recorded in global performance indices. Tracing is done by recording each of the observed events in a performance trace. Each record is composed of several parameters describing the event (see $\$ 3.2)$.

\subsection{Tracing and Behavioral Visualization}

Event driven monitoring is intensively used for performance debugging of parallel and distributed applications. Counting and timing are used to compute global performance indices whose evolution can sometimes be related to locations (procedures) [7] or execution phases [1] of the observed applications so that programmers can identify where and when the time goes. However, to reconstruct the behavior of the observed execution, more information is required and implies the use of execution traces. Traces can be used to reconstruct the dynamic behavior of an observed application so that the activity of processes, threads and processing elements and their communications can be checked along the execution time axis [12] (see figure 6).

\subsection{Versatile Visualization Tool}

Behavioral visualization tools include a trace-driven simulator to reconstruct the behavior of traced executions. Such simulators are usually dedicated to a given 
programming model. However, there exist various programming models that are used to program parallel or distributed applications [2|9 15 | 13 . In addition, these applications are often programmed using layered middleware systems; explaining performance problems may imply to observe their execution at several layers simultaneously, to relate a performance problem observed at one layer - MPI program for example — to the behavior of a lower abstraction layer — transport layer for example. Therefore it is necessary that behavioral visualization tools can be adapted to various programming models and able to reconstruct executions at several abstraction layers simultaneously.

\subsection{The Pajé Approach}

Pajé is a trace-driven behavioral visualization tool combining interactivity, scalability, extensibility and genericity properties [35. Interactivity allows users to move back and forth in time, to obtain more details on displayed objects or relate a visualization to a source program. Scalability is mainly provided by zooming functionalities in a fully interactive way so that application developers can zoom in from data aggregated along the space (groups of threads, of nodes) or time dimension to a detailed visualization of where the problem appears to be. Extensibility is the possibility of adding easily new functionalities to the tool; it is provided in Pajé by a careful modular design. Genericity is the faculty allowing users to adapt the Pajé visualization to various programming models by describing in the input data what they wish to visualize and how this should be done (see 44 ). Pajé could already be used in a variety of domains such as performance debugging of parallel [3] and distributed applications and monitoring of clusters [5]. The latest developments concern the production of execution traces which should be simple for all programming models that can be visualized with Pajé. The Tumit tool, described in the next section, allows tracing functions to be generated automatically as well as trace processing functions. The aim is to provide at least the same flexibility for monitoring as is provided by Pajé for visualization.

\section{Tumit}

The aim of Tumit is to help middleware or application developers to easily generate trace recording and trace manipulation functions. "Classical" tracing problems such as global dating or intrusion limitation and compensation [4] are not dealt with in this section.

\subsection{Software Tracing Instrumentation Techniques}

Instrumentation is the insertion of code to detect and record the application events. It can be done at several possible stages: in the source code of the traced application or at compile-time or by instrumenting the middleware used by the 
application or by direct instrumentation of compiled object code 4. Compiletime and object code instrumentation contradict the objective of being easy to adapt: the former requires to have access to the source code of the compiler and modify it while the latter is dependent on the operating system and the target platform. Instrumentation of communication libraries or runtime systems is not difficult for the developers of these middleware systems. However, if tracing functions are not included in the middleware, they have to be realized by the implementors of visualization tools or by their users; these people need to have access to the source codes of the middleware to be instrumented and also to update the instrumentation each time a new version of this middleware is released. For these reasons, it was decided not to realize another tracer for Pajé but instead to assist users in the development of ad hoc tracing facilities. From a description of the events to be traced, Tumit generates a set of tracing functions. Calls to these functions are inserted in the applications or middleware systems to be observed. Tumit also provides a set of data manipulation facilities to transform local into global dates or convert the monitored data from one format into another one (see \$3.2). To guarantee the maximum flexibility, format conversion functions are not directly provided but instead generated from a set of rewriting rules provided by the users (see $\$ 3.3$ ).

\subsection{Data Format}

A trace is composed of event records. Each record contains at least the following information: type of the event, (physical) date of the event and process identification of the process having performed the event. Some records contain additional parameters of the traced event such as receiver (sender) identification and message length in case of message emission (reception). Traces are usually recorded in a compact binary format to avoid memory buffer saturation and frequent disk accesses likely to alter the behavior of the observed application. They can be later processed - for example to correct the offsets and drifts of local clocks with respect to a global one [10] - , sorted and converted in the input data format of a visualization tool. The visualization tool input data format is a textual self defined data format (see [6] and \$4). In Pajé, trace format conversion is considered inevitable and therefore assisted by Tumit. Trace format conversion might involve more than coding the same data in a different way. Suppose for example that a blocking receive is recorded - by a "semantic-aware" tracer as a single event whose format includes two dates bounding the period when the emitting thread is blocked. When converting into the Pajé visualization input format, this event has to be transformed in two different visualization events, corresponding to the blocking and unblocking of the thread; the reason is that Pajé does not have notions of programming model semantics and has no notion of blocking receive. 


\subsection{Tumit Instrumentation Technique}

Tumit performs direct code instrumentation: the instructions generating the event records must be inserted in the programs before compilation (by the user of Pajé or by a preprocessor). Traced events are user-defined which provides complete flexibility in numbers and types of the recorded parameters. Tracing user-defined events requires specific functions to record these events and process the event records. In Tumit, these functions are automatically generated from events definitions. To illustrate the Tumit approach, we present examples extracted from the tracing and visualization of a small program using the Taktuk [11] communication library. Taktuk uses binomial tree topologies to launch parallel programs in logarithmic time on large-sized clusters of computers and to establish efficient communication networks. It can be seen as a communication library using two levels of parallelism: nodes and threads.

LEVEL (COM)
EVENT (PACK_MSG, int node, int thread) / Definition of the COM level
EVBNT (POST, int node, int thread, int dst, int stamp) // send
EVBNT (RECV, int node, int thread, int src, int stamp) // receive
EVENT (BND_UNPACK, int node, int thread) // end of receive

Fig. 2. Definition of events

Events definition. In this short example (see figure 2), four events are defined inside one level called сом: events are grouped in levels to ease the filtering of monitored data or allow users to mix traces recorded at different abstraction levels. For each event we define its name and for each user parameter, its name and type. The identification of the process (or thread) emitting the event has to be provided explicitly by the user since this identification is not standard, process or nodes in some cases, nodes and local thread identification in other cases. Stamps are used to match POST and RECV events.

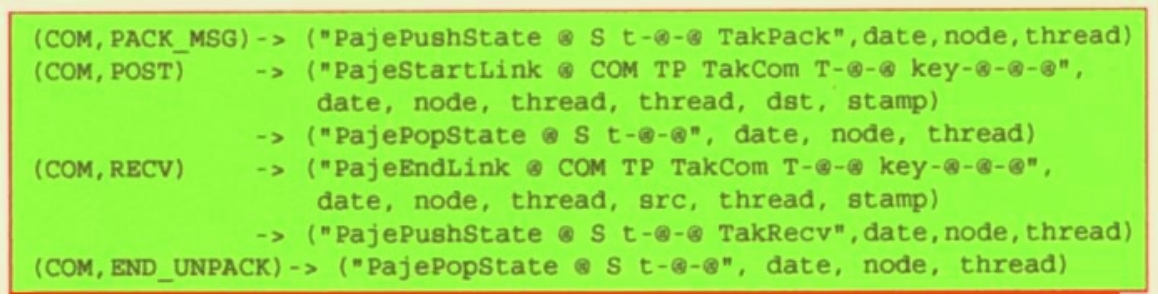

Fig. 3. Example Tumit conversion rules 


\begin{tabular}{|c|c|}
\hline \multicolumn{2}{|c|}{ // Keywords of the Pajé input data format are written in bold font. } \\
\hline PajeDefineContainerType P 0 "Prog" & // 0: no parent in type hieranchy \\
\hline PajeDefineContainerType N P "Node" & // There are nodes in a prog \\
\hline PajeDefineContainerType T N "Thread" & // There are threads in a node \\
\hline PajeDefineStateType S T "State" & // A thread has a State \\
\hline PajeDefineLinkType COM P T T "Comm" & // Com. from Thread to Thread \\
\hline \multicolumn{2}{|l|}{ // Nalue definition of previously defined entities } \\
\hline \multicolumn{2}{|c|}{ PajeDefineEntityValue TakWork $S$ "ThreadWorking" } \\
\hline \multicolumn{2}{|c|}{ PajeDefineEntityValue TakPack S "PackingTakCom" } \\
\hline \multicolumn{2}{|c|}{ PajeDefineEntityValue TakRecv S "ReceivingTakCom" } \\
\hline PajeDefineBntityValue TakCom COM "T & \\
\hline
\end{tabular}

Fig. 4. Definition of a hierarchy in a Pajé trace

Generation of tracing functions. From each event definition, Tumit generates a tracing function which can then be called from the user source code. The execution of a tracing function includes three phases: binding to a buffer and allocate another one if necessary; building an event record containing the parameters appearing in the events' definitions; if necessary, flushing the buffer to disk.

Generation of trace manipulation and conversion functions. Trace files need to be processed in order to be usable. Tumit generates functions allowing event records to be read (written) from (into) trace files or to be displayed under textual form. These functions are used in the implementation of more complex functionalities such as correcting the local time-stamps of the events to obtain globally coherent dates [10], sorting or filtering event trace files. As explained in 3.2 format conversion is often required. Tumit uses re-writing rules definitions to generate trace conversion functions. Figure 3 shows an example where these rules are used to transform monitoring data into the Pajé visualization tool format and produce the example of \$4. The output format is described as a character string where escape characters (@ sign) indicate substitutions by the recorded values of parameters. The corresponding parameters are written after the string. A rule with two arrows means that two events will be produced in the converted trace.

\section{Genericity of the Pajé Visualization Tool}

The Pajé visualization tool can be specialized for a given programming model. The visualization to be constructed from the traces can be described by the user, provided that the types of the objects appearing in the visualization are hierarchically related and that this type hierarchy can be described as a tree 
(see figures 4 and [6]. The Pajé self-defined input data format [5] is based on two generic types: containers and entities. While entities can be considered as elementary visual objects, containers are complex objects, each including a (potentially high) number of entities. An entity type is further specified by one of the following generic types: event, state, link or variable. Each entity also has a value, which allows its identification among other entities of the same type. In the example of figure 4, three hierarchically related containers are defined, named Prog, Node and Thread. The Thread container is composed of entities of type $\mathrm{S}$ or ThreadState of the generic type "state" and will therefore be represented as rectangles showing the successive states of the thread. These states may have three possible values, ThreadWorking or PackingTakCom or ReceivingTakCom. Another entity type named Comm of type "link", connecting threads, is defined and will be represented as an arrow with a source and destination.

Monitoring data, recorded during the execution of a small Taktuk program, using trace functions generated by Tumit from the event definitions of $\$ 3.3$. was converted, using the rewrite rules presented in $\$ 3.3$, in the Pajé data format defined above. A small excerpt of this Pajé file is given in figure 5 it exemplifies the creation of two threads and the sending of a message from one thread to the other. The identification of each thread is obtained by concatenation of a global node identification with a thread identification local to the node.

\begin{tabular}{|c|c|}
\hline PajeCreateContainer 0.00 TP P 0 "Test Prog" & // Monitored program \\
\hline PafeCreateContainer $0.00 \mathrm{~N} 1 \mathrm{~N}$ TP "Node 1 " & // Node 1 created \\
\hline PajeCreateContainer 0.00 N2 N TP "Node 2" & // Node 2 created \\
\hline PajeCreateContainer 0.12 T-1-1 T N1 "Thread-1- & "// Creation of thread-1-1 \\
\hline PajeSetSate 0.12 S T-1-1 TakWork & // T-I-I initial state \\
\hline PajeCreateContainer 0.15 T-2-1 T N1 "Thread-2- & "// Creation of thread-2-1 \\
\hline PajeSetSate 0.15 S T-2-1 TakWork & // T-2-1 initial state \\
\hline PajePushstate 0.25 S T-1-1 TakPack & // T-1-I packs message \\
\hline PajestartLink 0.42 COM TP TakCom T-1-1 key121 & // T-1-I sends message \\
\hline Pajepopstate 0.42 S T-1-1 & // T-1-1 back to work \\
\hline 0.55 COM TP TakCom T-2-1 key121 & // T-2-1 receives msg \\
\hline PajePushstate 0.55 S T-2-1 TakRecv & // $T-2-1$ changes state \\
\hline PajePopstate 0.75 S T-2-1 & // T-2-1 back to work \\
\hline
\end{tabular}

Fig. 5. Excerpt of converted trace file

\section{Related Work}

VampirTrace and Vampir [12] allow users to obtain easily behavioral visualizations of parallel programs executions. However these tools are bound to a programming model such as MPI and cannot be easily adapted. Tau [14 is a very flexible and portable performance monitoring tool which records information using mainly the counting and timing techniques (see \$2.1). The tracing facility which should be provided through the definition of "user events" was not yet 

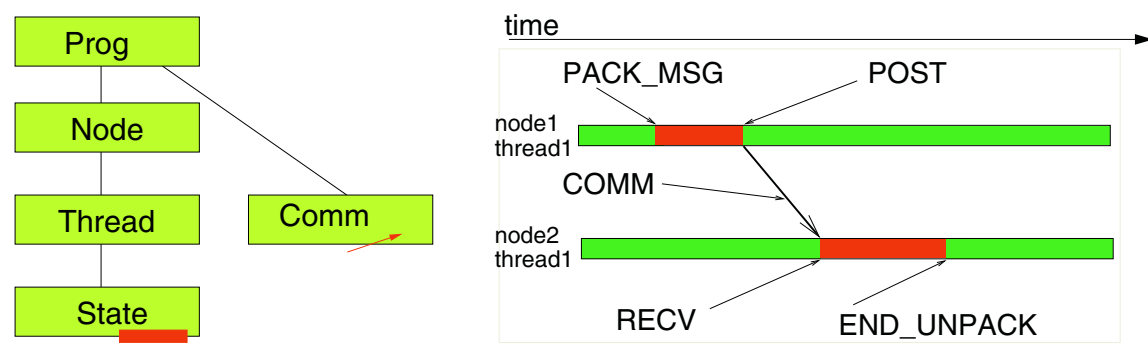

Fig. 6. Simple Pajé type hierarchy and visualization of Taktuk communication events

available in Tau at the time Tumit was designed. In the Paraver visualization and analysis tool [8], users can assign semantics — within a programming model paradigm - to the types of event records to indicate how trace file should be visualized.

\section{Conclusion and Future Work}

The Pajé approach for performance debugging is based on event tracing and behavioral visualizations of the observed executions. One of the objectives of Pajé is to face the multiplicity of parallel and distributed programming models. From a description of events to be traced, Tumit generates event recording functions and trace manipulation programs. The Pajé visualization tool can be adapted to various programming models. Future work includes experimenting with multi-level tracing and visualizations - mixing hardware counters, thread management, communications and high level parallel programming model — or adapting global clock implementation techniques to scale to hundreds of processing elements or more.

Acknowledgements. Jacques Briat suggested several of the proposed techniques. This work is partly supported by INRIA Rhône-Alpes via the LIPS project (joint project of INRIA and BULL).

\section{References}

1. B. Buck and J. K. Hollingsworth. An API for runtime code patching. Journal of SuperComputing Applications and High Performance Computing, 14(4):317-329, Winter 2000.

2. R. Chandra et al. Parallel Programming in OpenMP. Morgan Kaufmann, 2000.

3. J. Chassin de Kergommeaux, B. de Oliveira Stein, and P. Bernard. Pajé, an interactive visualization tool for tuning multi-threaded parallel applications. Parallel Computing, 26(10):1253-1274, aug 2000. 
4. J. Chassin de Kergommeaux, E. Maillet, and J.-M. Vincent. Monitoring parallel programs for performance tuning in cluster environments. In J. Cunha et al., editors, Parallel Program Development for Cluster Computing, volume 5, chapter 6, pages 131-150. Nova Science, 2001.

5. J. C. de Kergommeaux and B. de Oliveira Stein. Flexible performance visualization of parallel and distributed applications. Future Generation Computer Systems, 19(5):735-748, 2003.

6. B. de Oliveira Stein, J. Chassin de Kergommeaux, and G. Mounié. Pajé trace file format. Technical report, ID-IMAG, Grenoble, France, 2002. http://wwwid.imag.fr/Logiciels/paje/publications.

7. L. DeRose and D. Reed. Svpablo: A multi-language architecture-independent performance analysis system. In Procs of the International Conference on Parallel Processing (ICPP'99), pages 311-318, Fukushima, Japan, September 1999.

8. European Center for Parallelism of Barcelona, http://www.cepba.upc.es/paraver. Paraver: Parallel Program Visualization and Analysis Tool, 2001.

9. M. P. I. Forum. MPI: A message passing interface standard. International Journal of Supercomputer Applications, 8(3/4), 1994.

10. É. Maillet and C. Tron. On Efficiently Implementing Global Time for Performance Evaluation on Multiprocessor Systems. Journal of Parallel and Distributed Computing, 28:84-93, 1995.

11. C. Martin and O. Richard. Parallel launcher for cluster of PC. In Proceedings of ParCo 2001. Imperial College Press, London, 2001.

12. W. Nagel et al. VAMPIR: Visualization and analysis of MPI resources. Supercomputer, 12(1):69-80, 1996.

13. A. Pope. The CORBA reference guide. Addison Wesley, 1998.

14. S. Shende, A. D. Malony, et al. Portable profiling and tracing for parallel scientific applications using C++. In Proc. of SPDT'98: ACM SIGMETRICS Symposium on Parallel and Distributed Tools, pages 134-145, Aug. 1998.

15. Sun Microsystems Inc., http://java.sun.com/docs/books/tutorial/. The Java(tm) Tutorial, 2003. 